\title{
First description of a giant spindle-cell myxoid tumor with an FGFR1-CAPRIN1 fusion on the back of an infant's hand
}

\author{
Raphael Staubach ${ }^{1}$, Helen Glosse ${ }^{2}$, Christian Vokuhl ${ }^{3}$, Oliver Diez ${ }^{2}$, Ewa Koscielniak ${ }^{2}$, and \\ Steffan Loff ${ }^{4}$ \\ ${ }^{1}$ Klinikum Stuttgart \\ ${ }^{2}$ Klinikum Stuttgart Olgahospital Frauenklinik \\ ${ }^{3}$ University of Kiel \\ ${ }^{4}$ Pediatric Surgery
}

October 26, 2020

\begin{abstract}
In this article we report a case of a huge, fast growing, spindle cell myxoid tumor on the back of an infant's hand with a previously undescribed FGFR1-CAPRIN1 fusion. We discussed here difficulties in clinical decision making in rare cases of life quality threatening soft tissue tumors in infants.
\end{abstract}

\section{Introduction:}

Soft tissue sarcomas are very rare in infancy with 8-10\% of all neoplasias (1). The incidence of all soft tissue sarcomas in children under one year of age is about 1.6 per 100.000 per year (2). They can occur anywhere in the body and are of mesenchymal origin (3). The most common location is described in the extremities (4).

\section{Case representation:}

After birth a nodular change on the back of the right hand was observed in a male infant. In the following two months, there was a clear progression of the findings, so that at the age of 8 weeks, an MRI was first performed and then a test excision. At this time the lesion already measured $4.7 \mathrm{~cm} \times 3.2 \mathrm{~cm} \mathrm{x} 4.3 \mathrm{~cm}$ on the MRI. The histopathological evaluation was difficult, however in the reference pathology, a benign spindle cell lesion without a CTNNB1 mutation or break in the NTRK1-3 genes was diagnosed. After further rapid progression of the tumor occurred, the child was referred to a Department of Pediatric Oncology clinic, where the lesion was deemed non-resectable. After consultation with the CWS (Cooperative Weichteilsarkomstudiengruppe) study center, chemotherapy was advised according to the recommendations for the treatment of fibromatosis. The parents refused chemotherapy.

After another four weeks, an enormous increase in the size of the tumor leading to an ulceration had evolved (Fig.1). The function of the whole right extremity was completely impaired due to the weight of the lesion. The neurologic function was as far as assessable not deficient. A decision to perform the best possible resection in a function-preserving manner was taken after a multidisciplinary discussion with the CWS tumor board. Prior to the operation, the total body MRI showed a tumor measuring $2 \mathrm{~cm} \times 6 \mathrm{~cm} \times 9.5 \mathrm{~cm}$ $(342 \mathrm{ml})$ with direct contact of the tumor with the extensor tendons, vessels and nerves as well as a close relationship to the metacarpalia in interdigital space I and II. No distant metastatic lesions were found. The excision of the tumor was performed at the age of 6 months. Intraoperatively, a very precise preparation was required to preserve the function of the hand (Fig.2). The tumor was removed in total and the large wound 
area with the preserved skin margins were closed. Postoperatively, the motor function of the arm and hand regained normal function (Fig.3).

The weight of the tumor was 999.5g (Fig.2). An imaging two and three months after resection showed a small $(1 \mathrm{~cm})$ residual lesion between metacarpale I and metacarpale II, which could be resected with acceptable margins $(0.3 \mathrm{~cm}, 0.2 \mathrm{~cm}$ and $0.5 \mathrm{~cm})$. This was only possible by taking along the periosteum of metacarpale II, the periosteum and part of the base of metacarpale I, part of the M. adductor and the tendon sheaths of the extensor tendons D1 and motor function, blood circulation and sensitivity were again unimpaired postoperatively.

The child had an inconspicuous postoperative course and normal function of the hand.

\section{Histology:}

The histopathological result of the biopsy and the first resection showed a spindle-cell, partly myxoid tumor. The tumor cells are repeatedly arranged in trains or bundles, also in a vertebral arrangement. Cells had ovoid to elongated nuclei with moderate nuclear hyperchromasia and overall low nuclear pleomorphism. The tumor reached the ink-marked resection margin in several places. Immunohistochemically, there was a clear positivity for CD34 and FGFR1-CAPRIN1 fusion which was detected by RNA panel sequencing. The fusion described above has not been described in the literature so far. The tumor was classified as a spindle-cell tumor with intermediate malignancy.

Histology of the second surgery showed similar findings.

\section{Discussion:}

Soft tissue tumors and sarcomas in infancy are very rare. The congenital tumors could have an enormous growth tendency.

In this case, a delayed presentation of the child in a Department of Pediatric Oncology and difficulties in a histologic assessment of a biopsy sample delayed the surgery until the age of 6 months when the tumor had already reached a grotesque size.

The proposal to perform chemotherapy was based on the experience of other benign and intermediate malignant tumor in infancy which may show very different clinical courses. Therefore, it was very difficult to assess the expected effectiveness of chemotherapy in this rare entity. In addition, the tumor regression, if at all, could be expected to be very slow, so that the cumulative doses of the applied cytostatic drugs would be considerably high, which is unfavorable for infants. Cyclophosphamide, Actinomycin D or Vincristine were discussed here (8).

Another important factor is the motor development of the arm and functionality of the hand, which in the case of a 1-kilogram tumor was massively impaired. For this reason, from an oncological point of view, a non-radical resection was acceptable (5).

Radiotherapy for this type of tumor would be possible in principle but is not indicated in infancy due to its age and severe late sequelae such as possible cartilage and bone deformities (6). Earlier surgery would have increased the probability of primary R0 resection and reduced the probability of possible metastasis. However, we consider the R1 resection achieved after initial surgery without neoadjuvant therapy to be acceptable and the only option due to the size of the tumor at the time of excision. The complication-free second resection with acceptable tumor-free margins and complete functional preservation of the affected extremity support our therapeutic decision. The last MRI control 9 months after the second resection showed no renewed tumor growth.

\section{Conclusion:}

1. Children with unclear lump should be referred for further diagnostic to specialized centers of pediatric oncology. 
2. A reference pathology with molecular characterization is necessary.

3. The discussion of therapeutic decisions must always be interdisciplinary in view of the goal, taking into account the age, localization and biology of the tumor and taken by experts in the field of pediatric solid tumors.

4. Comparison with previously published data is limited because the histological diagnoses are outdated without the molecular genetics we cannot reflect on the heterogeneity and the biology of related tumors $(7 ; 9)$.

\section{keywords:}

sarcoma, wide margin resection, neonatal, pediatric surgery

Funding: None. Conflicts of interest: None declared. Ethical approval: Not required.

\section{References:}

1. Orbach D, Sarnacki S, Brisse HJ, Gauthier-Villars M, Jarreau PH, Tsatsaris V, et al. Lancet Oncol. 2013 Dec;14(13)

2. Sultan I, Casanova M, Al-Jumaily U, Meazza C, Rodriguez-Galindo C, Ferrari A. Soft tissue sarcomas in the first year of life.

3. Eur J Cancer. 2010 Sep; 46(13): 2449-56.von Mehren M, Lor Randall R, DeLaney T, George S, Riedel R, Scheutze S, et al. NCCN Clinical Practice Guidelines in Oncology (NCCN Guidelines@) Soft Tissue Sarcoma. (2014;2.2014:126)

4. Tran QN, Kim AC, Gottschalk AR, Wara WM, Phillips TL, O'Donnell RJ, et al. Clinical outcomes of intraoperative radiation therapy for extremity sarcomas. Sarcoma. 2006;2006(1):91671. Pubmed Central PMCID: 1557794, Epub (2006/10/17)

5. Fernandez-Pineda I, Neel MD, Rao BN. Current management of soft tissue sarcoma and Benign Tumors with Local Aggresiveness

6. Laskar, S., Pilar, A., Khanna, N., Puri, A., Gulia, A., Qureshi, S., Ramadwar, M. Interstitial brachytherapy for pediatric soft tissue sarcoma: Evolving practice over three decades and long-term outcomes. Pediatric Blood \& Cancer (2018)

7. Orbach D, Rey A, Oberlin O, et al. Soft tissue sarcoma or malignant mesenchymal tumors in the first year of life: experience of the International Society of Pediatric Oncology (SIOP) Malignant Mesenchymal Tumor Committee. Journal of clinical oncology: official journal of the American Society of Clinical Oncology 2005;23:4363-71.

8. Koscielniak E, Harms D, Schmidt D, et al. Soft tissue sarcomas in infants younger than 1 year of age: a report of the German Soft Tissue Sarcoma Study Group (CWS-81). Medical and pediatric oncology 1989;17:105-10.

9. Spunt SL, Million L, Chi YY, et al. A risk-based treatment strategy for non-rhabdomyosarcoma softtissue sarcomas in patients younger than 30 years (ARST0332): a Children's Oncology Group prospective study. The Lancet Oncology 2020;21:145-61. 

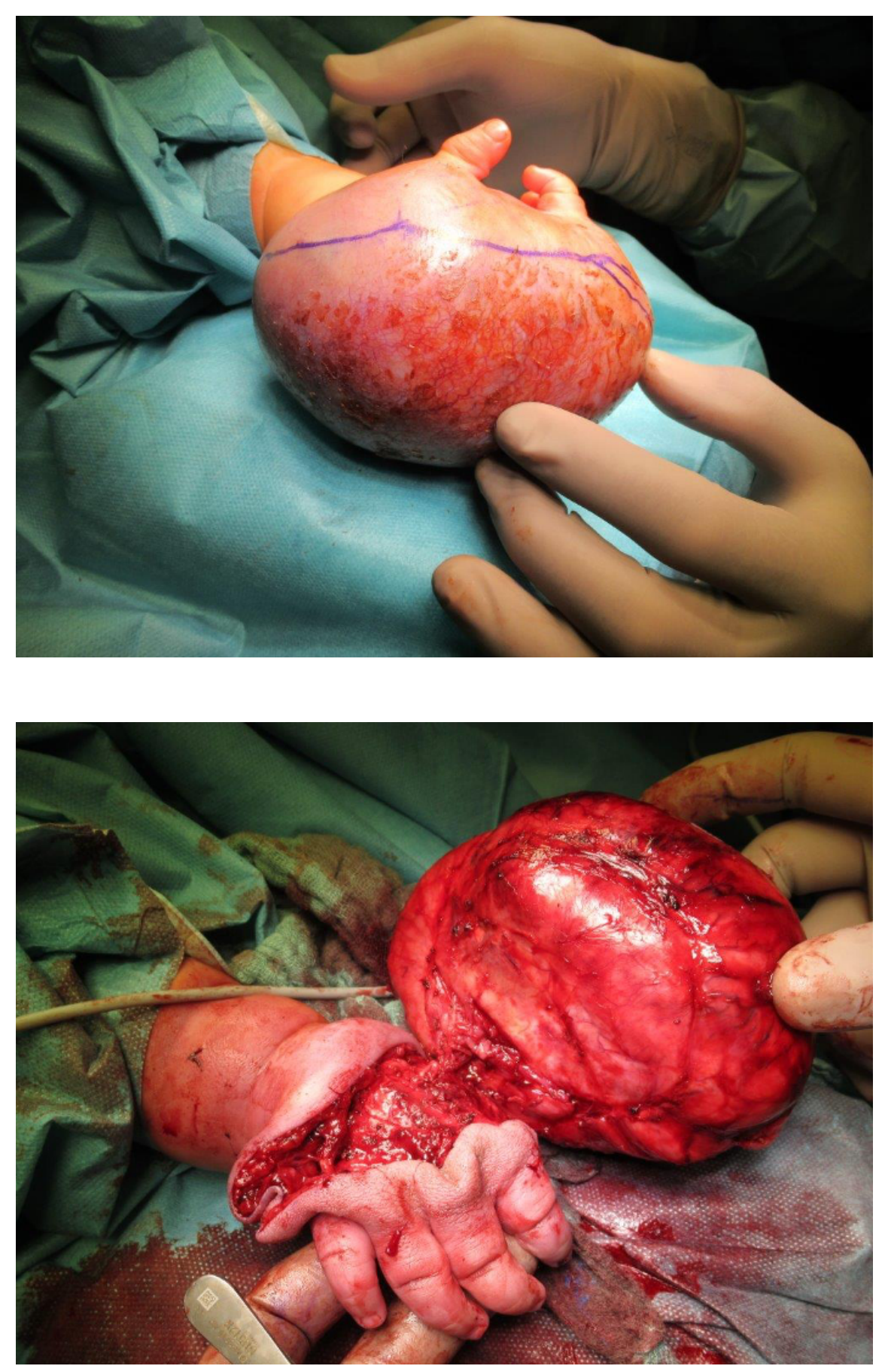


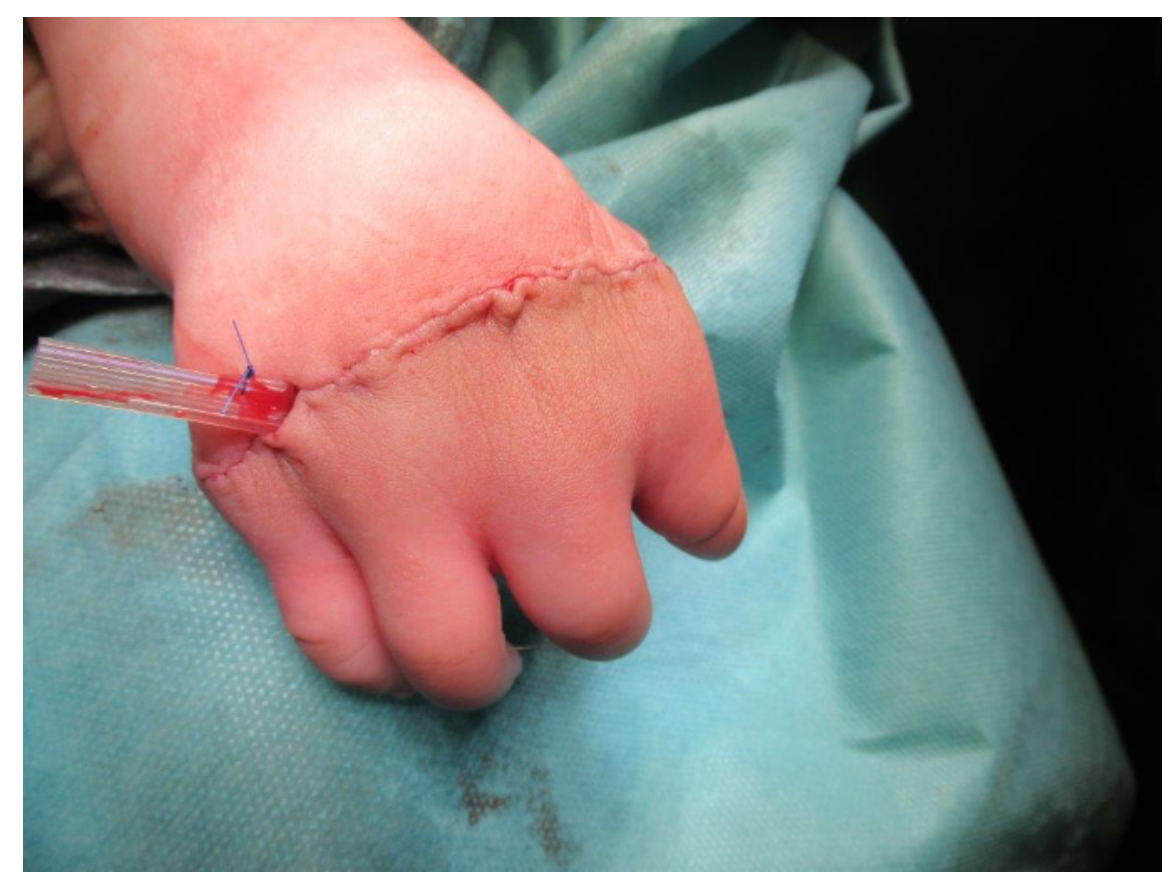

